Article

\title{
Serum Mac-2 Binding Protein Levels Associate with Metabolic Parameters and Predict Liver Fibrosis Progression in Subjects with Fatty Liver Disease: A 7-Year Longitudinal Study
}

\author{
Yoshihiro Kamada ${ }^{1,2}{ }^{\oplus}$, Koichi Morishita ${ }^{1}$, Masahiro Koseki ${ }^{3}$, Mayu Nishida ${ }^{1}$, Tatsuya Asuka ${ }^{1}$, \\ Yukiko Naito ${ }^{1}$, Makoto Yamada ${ }^{4}$, Shinji Takamatsu ${ }^{1}{ }^{1}$, Yasushi Sakata ${ }^{3}$, Tetsuo Takehara ${ }^{2}$ and \\ Eiji Miyoshi ${ }^{1, *}$ (D) \\ 1 Department of Molecular Biochemistry and Clinical Investigation, Osaka University Graduate School of \\ Medicine, Suita, Osaka 565-0871, Japan; ykamada@gh.med.osaka-u.ac.jp (Y.K.); \\ a.xxivk.m@gmail.com (K.M.); nmayu.1119@gmail.com (M.N.); tasuka25@gmail.com (T.A.); \\ rin.hana.ren@gmail.com (Y.N.); shinjit@sahs.med.osaka-u.ac.jp (S.T.) \\ 2 Department of Gastroenterology and Hepatology, Osaka University Graduate School of Medicine, Suita, \\ Osaka 565-0871, Japan; takehara@gh.med.osaka-u.ac.jp \\ 3 Department of Cardiovascular Medicine, Osaka University Graduate School of Medicine, Suita, \\ Osaka 565-0871, Japan; koseki@cardiology.med.osaka-u.ac.jp (M.K.); \\ yasushisk@cardiology.med.osaka-u.ac.jp (Y.S.) \\ 4 aMs NEW OTANI CLINIC, Osaka 540-0001, Japan; kazuaki.sakata@ams-group.jp \\ * Correspondence: emiyoshi@sahs.med.osaka-u.ac.jp; Tel.: +81-6-6879-2590
}

Received: 12 May 2020; Accepted: 10 June 2020; Published: 12 June 2020

\begin{abstract}
Background: Mac-2 binding protein (M2BP) is a highly glycosylated secreted glycoprotein that is involved in immune defense and regulation. Our cross-sectional studies indicated that serum M2BP was a useful liver fibrosis biomarker for nonalcoholic fatty liver disease (NAFLD). In this study, we conducted a 7-year longitudinal study to investigate the significance of serum M2BP levels (baseline and at 7-year follow-up) and their relationships with other metabolic parameters of fatty liver disease. Methods: We enrolled 715 study subjects (521 male and 194 female) during health examinations. Study subjects received blood sampling tests and abdominal ultrasound tests at baseline and follow-up. Results: Univariate analyses demonstrated that serum M2BP levels were significantly correlated with various parameters related to metabolic risk (body mass index (BMI), systolic blood pressure, triglyceride, high density lipoprotein (HDL)-cholesterol) and metabolic syndrome diseases (obesity, hypertension, dyslipidemia, diabetes mellitus, fatty liver (FL)). Multiple logistic regression analyses demonstrated that BMI and FL were independent determinants for serum M2BP levels. Baseline serum M2BP levels were significant independent determinants for changes in platelet count, Fibrosis-4 (FIB4) index, and NAFLD fibrosis score. Higher serum M2BP levels $(>1.80 \mu \mathrm{g} / \mathrm{mL})$ strongly correlated with changes in the FIB4-index. Conclusions: The results of this study suggest that changes in serum M2BP levels reflect changes in specific metabolic disease-related parameters, and baseline serum M2BP levels could predict changes in liver fibrosis.
\end{abstract}

Keywords: Mac-2 binding protein; fatty liver; metabolic syndrome; liver fibrosis; biomarker; longitudinal study 


\section{Introduction}

Nonalcoholic fatty liver disease (NAFLD) is one of the most common causes of chronic liver disease and a growing medical problem worldwide [1]. A wide spectrum of hepatic histological changes occur in NAFLD patients, ranging from the generally nonprogressive nonalcoholic fatty liver to nonalcoholic steatohepatitis (NASH). It is important to assess the degree of liver fibrosis during the clinical progression of NAFLD to predict disease progression and formulate therapeutic management decisions [2,3]. Liver biopsy remains the gold standard for assessing liver fibrosis [4,5]. However, liver biopsy has significant limitations such as pain, risk of severe complications, sampling error [6], cost [7], and patient unwillingness to undergo invasive testing. A recent study reported that liver fibrosis was independently associated with long-term outcome in NAFLD patients [8]. Therefore, there is an urgent need for a reliable and noninvasive test that accurately assesses the degree of liver fibrosis. Recently, novel noninvasive approaches such as transient elastography (e.g., FibroScan, acoustic radiation force impulse), magnetic resonance elastography (MRE), and various scoring systems (e.g., Fibrosis (FIB)-4 index, NAFLD fibrosis score) can be used to measure the liver fibrosis severity in patients with chronic liver disease [9-12]. However, distinguishing early liver fibrosis from non-fibrotic liver is difficult using transient elastography. Various scoring systems and biomarkers for measuring liver fibrosis severity are available [13-15], but few individual systems or biomarkers have clinical validity. Therefore, there is still the need for a reliable liver fibrosis biomarker.

Mac-2 binding protein (M2BP) is a glycoprotein that contains seven potential N-glycosylation sites $[16,17]$. We previously identified M2BP as one of the major fucosylated glycoproteins that were secreted from the HuCCT-1 liver bile duct cancer cell line [18]. Serum M2BP concentrations increase in patients with various cancers (e.g., pancreatic, breast, and lung cancer), viral hepatitis, and autoimmune disease [16]. M2BP is essentially undetectable in normal liver, but is easily detected in hepatocytes from patients with chronic hepatitis type $\mathrm{C}(\mathrm{CHC})$ during the progression of liver fibrosis $[19,20]$. Previously, we developed an enzyme-linked immunosorbent assay (ELISA) kit to measure serum total M2BP levels in human [18].

We recently observed that serum M2BP levels could be used to predict the histological severity of hepatic fibrosis in NAFLD patients [18,21]. Wisteria floribunda agglutinin (WFA)-positive M2BP ( $\mathrm{WFA}^{+}-\mathrm{M} 2 \mathrm{BP}$ ), also known as M2BPGi (Mac-2 Binding Protein Glycosylation isomer), is a novel serum fibrosis biomarker for $\mathrm{CHC}$ [22]. This biomarker distinguishes the glycan structure of WFA-detectable M2BP; it was developed using a glycan-based immunoassay for assessing liver fibrosis severity in CHC patients [22]. WFA ${ }^{+}-\mathrm{M} 2 \mathrm{BP}$ also can be used for assessing the liver fibrosis stage in NAFLD patients [23]. Our recent meta-analysis suggested that serum $\mathrm{WFA}^{+}-\mathrm{M} 2 \mathrm{BP}$ was a reliable biomarker that detected advanced fibrosis in various chronic liver diseases [24]. However, the liver fibrosis prediction abilities for the other chronic liver diseases (NAFLD, chronic hepatitis type B, primary biliary cirrhosis, and autoimmune hepatitis) are relatively lower than that for CHC [22,23,25-27]. Previously, our study also revealed that compared with $\mathrm{WFA}^{+}-\mathrm{M} 2 \mathrm{BP}$, total M2BP measured by our developed ELISA had a greater ability to predict NAFLD fibrosis stage [28]. In this study, we measured serum total M2BP levels in our study subjects.

These combined results indicate that serum M2BP levels are useful liver fibrosis biomarkers. However, the relationships among serum M2BP levels, metabolic parameters, and liver fibrosis progression have not been investigated in a longitudinal study. The objective of this study was to examine the relationships among changes in serum M2BP levels, changes in metabolic parameters, and changes in liver fibrosis markers using a 7-year follow-up study. Recent studies reported that alcohol and obesity synergistically sensitize liver damage $[29,30]$. The concept of metabolic dysfunction-associated fatty liver disease (MAFLD) was proposed recently [31]. The criteria for MAFLD regardless of alcohol consumption are based on evidence of fatty liver along with one of the following three parameters: overweight/obesity, presence of type 2 diabetes mellitus (DM), or evidence of metabolic dysregulation. Our present study investigated MAFLD subjects and did not exclude subjects with a history of alcohol abuse. 


\section{Patients and Methods}

\subsection{Ethical Committee Approval}

The research and informed consent protocols were approved for use in a multicenter study by the institutional review boards at Osaka University Hospital and aMs NEW OTANI CLINIC. Written informed consent was obtained from each subject at time of enrollment at each institute. The study was conducted in accordance with the Helsinki Declaration.

\subsection{Subjects in Medical Health Check-Ups}

A total of 2167 individuals who underwent a health examination at aMs NEW OTANI CLINIC (Osaka, Japan) from 2009 to 2011 were initially recruited into the study, and 806 of these subjects received a follow-up health examination after 7 years. No specific inclusion criteria were applied. The following exclusion criteria were applied: history of hepatic disease such as CHC or concurrent active hepatitis B (seropositive for hepatitis B surface antigen), autoimmune hepatitis, primary biliary cholangitis, sclerosing cholangitis, hemochromatosis, $\alpha 1$-antitrypsin deficiency, Wilson's disease, and hepatic injury caused by substance abuse excluding alcohol consumption. Alcohol consumption (g/week) was calculated based on the self-response questionnaire sheets at medical check-ups. Subjects receiving anticoagulant therapy, which could affect platelet measurements, were also excluded. A total of 715 subjects (521 male and 194 female) received an abdominal ultrasound test. The diagnosis of fatty liver was based on the results of the abdominal ultrasound examination performed by trained technicians. A fatty liver was defined as a liver parenchyma with an echogenicity greater than that of the kidney cortex, the presence of vascular blurring, and deep attenuation of the ultrasound signal [32,33]. Blood serum was collected from each subject during the health examination and was stored at $-80^{\circ} \mathrm{C}$ until analysis.

\subsection{Anthropometric and Laboratory Evaluation}

Anthropometric variables (height and weight) were measured while each subject was in the standing position. Body mass index (BMI) was calculated as weight $(\mathrm{kg})$ divided by the square of height in meters $\left(\mathrm{m}^{2}\right)$. Systolic and diastolic blood pressure (SBP and DBP) values were measured (to the nearest $\mathrm{mm} \mathrm{Hg}$ ) while each subject was in the sitting position. Serum biochemical variables [aspartate aminotransferase (AST), alanine aminotransferase (ALT), $\gamma$-glutamyltransferase (GGT), total bilirubin (T-Bil), creatinine, choline esterase (CHE), total cholesterol (T-Chol), triglyceride (TG), high density lipoprotein cholesterol (HDL-C), low density lipoprotein cholesterol (LDL-C), uric acid, iron, fasting blood glucose (FBG), albumin, and platelet count] were measured using a conventional automated blood analyzer. The FIB4-index (based on subject age, serum AST and ALT levels, and platelet counts) was calculated for each subject as reported previously [age $\times$ AST $(\mathrm{U} / \mathrm{L}) /$ Platelet count $\left(\times 10^{9} / \mathrm{L}\right) / \sqrt{ }$ ALT $\left.(\mathrm{U} / \mathrm{L})\right][34,35]$. Impaired fasting glucose (IFG) was defined as an FBG of 110-125 mg/dL. The NAFLD fibrosis score (NFS) was calculated for each of the subjects as previously reported $\left[1.675+0.037 \times\right.$ age $($ years $)+0.094 \times \mathrm{BMI}\left(\mathrm{kg} / \mathrm{m}^{2}\right)+1.13 \times \mathrm{IFG} / \mathrm{DM}($ yes $=1$, no $=0)+0.99 \times$ AST/ALT $-0.013 \times$ platelet count $\left(\times 10^{9} / \mathrm{L}\right)-0.66 \times$ albumin $\left.(\mathrm{g} / \mathrm{dL})\right]$ [12]. We used an ELISA kit (Immuno-Biological Laboratory Co. Ltd., Fujioka, Japan, code \# 27362) to measure serum M2BP [18]. Changes in various parameters were defined as follows: ((parameter data at follow-up) - (parameter data at baseline)).

\subsection{Diagnostic Criteria for Metabolic Syndrome-Related Diseases}

Diagnostic criteria for metabolic syndrome-related diseases were as follows: obesity [BMI $\geq 25$ $(n=310,43.3 \%)$ ]; hypertension [history of drug use, $\mathrm{SBP} \geq 130$, or DBP $\geq 85(n=250,34.9 \%)$ ]; dyslipidemia [history of drug use, TG $\geq 150$, and/or HDL-C $<40(n=279,39.0 \%)$ ]; and diabetes mellitus [history of drug use, $\mathrm{FBG} \geq 126$, or $\mathrm{HbA} 1 \mathrm{c} \geq 6.5 \%(n=428,59.8 \%)]$. There were no significant differences among subjects with or without drug use (antihypertensive drug, dyslipidemia drug) in AST and ALT values. 


\subsection{Statistical Analysis}

Statistical analyses were performed using JMP Pro 14.0 software (SAS Institute Inc., Cary, NC, USA). Results were expressed as mean \pm standard deviation (SD). The statistical analysis included descriptive statistics, analysis of variance, the Wilcoxon test, and the Pearson test. Chi-square tests were used for categorical factors. The AST, ALT, GGT, TG, FBG, HbA1c, M2BP, and FIB4-index values did not display Gaussian distributions; therefore, these parameters were common log-transformed before analysis. Multivariate logistic regression analyses were performed to identify significant determinants. Differences were considered as statistically significant at $p<0.05$.

\section{Results}

\subsection{Characteristics of the Study Participants}

The results of the clinical and biochemical analyses of individuals in the study population are presented in Table 1. Among the 715 study subjects, 442 were diagnosed with fatty liver disease at baseline using abdominal ultrasound, and 423 subjects were diagnosed with fatty liver disease at follow-up. Among 442 fatty liver subjects at baseline, 382 subjects (86.4\%) were still diagnosed with fatty liver at follow-up. The ratio of subjects with fatty liver disease at follow-up was significantly lower than that at baseline. The ratio of males in the group with fatty liver disease (FL+) was greater than the ratio of males in groups without fatty liver disease (FL-) at both baseline and follow-up. BMI was greater in subjects with fatty liver disease. Alcohol consumption (g/week) was not different between (FL+) and (FL-) at baseline, but significantly higher in (FL+) than in (FL-) at follow-up. The serum levels of AST, ALT, GGT, albumin, CHE, TG, LDL-C, uric acid, and FBG were significantly higher in FL+ subjects than in FL- subjects at both baseline and follow-up. The FIB4 index was lower in FL+ subjects than in FL- subjects. The NFS was not different between (FL+) and (FL-) at baseline, but significantly higher in (FL+) than in (FL-) at follow-up. Serum HDL-C levels were lower in FL+ subjects. Serum M2BP levels were higher in FL+ subjects at both baseline and follow-up.

Table 1. Clinical and biochemical parameters of study subjects.

\begin{tabular}{|c|c|c|c|c|c|c|}
\hline \multirow{2}{*}{ Variable } & \multicolumn{3}{|c|}{ Baseline Parameters } & \multicolumn{3}{|c|}{ Follow-Up Parameters after 7 Years } \\
\hline & Fatty Liver (-) & Fatty Liver (+) & $p$ Value * & Fatty Liver (-) & Fatty Liver (+) & $p$ Value * \\
\hline Number of study subjects & 273 & 442 & & 292 & 423 & $<0.0001^{\#}$ \\
\hline Age (years) & $54.0 \pm 8.3$ & $53.5 \pm 6.8$ & N.S. & $61.4 \pm 8.7$ & $59.8 \pm 6.3$ & $<0.05$ \\
\hline Gender $(\mathrm{M} / \mathrm{F})$ & $155 / 118$ & $366 / 76$ & $<0.0001$ & $175 / 117$ & $346 / 77$ & $<0.0001$ \\
\hline $\operatorname{BMI}\left(\mathrm{kg} / \mathrm{m}^{2}\right)$ & $22.0 \pm 2.7$ & $26.3 \pm 3.8$ & $<0.0005$ & $21.9 \pm 2.8$ & $26.0 \pm 3.8$ & $<0.0001$ \\
\hline Alcohol consumption (g/week) & $86.6 \pm 112.6$ & $109.3 \pm 128.5$ & N.S. & $91.0 \pm 123.5$ & $114.1 \pm 132.2$ & $<0.05$ \\
\hline SBP $(\mathrm{mm} \mathrm{Hg})$ & $110.4 \pm 14.3$ & $120.0 \pm 15.4$ & $<0.0001$ & $106.8 \pm 15.8$ & $114.2 \pm 14.2$ & $<0.0001$ \\
\hline AST (U/L) & $24.3 \pm 13.1$ & $32.1 \pm 15.4$ & $<0.0001$ & $23.2 \pm 10.2$ & $30.4 \pm 18.3$ & $<0.0001$ \\
\hline $\operatorname{ALT}(\mathrm{U} / \mathrm{L})$ & $23.4 \pm 17.4$ & $43.8 \pm 24.8$ & $<0.01$ & $19.5 \pm 10.0$ & $35.7 \pm 23.4$ & $<0.0001$ \\
\hline GGT (U/L) & $50.8 \pm 85.2$ & $74.0 \pm 78.7$ & $<0.0001$ & $42.3 \pm 62.3$ & $62.8 \pm 66.5$ & $<0.0001$ \\
\hline T-Bil (mg/dL) & $0.79 \pm 0.32$ & $0.80 \pm 0.30$ & N.S. & $0.80 \pm 0.30$ & $0.86 \pm 0.34$ & $<0.05$ \\
\hline Albumin (g/dL) & $4.23 \pm 0.24$ & $4.40 \pm 0.22$ & $<0.0001$ & $4.33 \pm 0.23$ & $4.46 \pm 0.23$ & $<0.0001$ \\
\hline Creatinine $(\mathrm{mg} / \mathrm{dL})$ & $0.78 \pm 0.16$ & $0.83 \pm 0.16$ & $<0.0001$ & $0.80 \pm 0.18$ & $0.87 \pm 0.30$ & $<0.0001$ \\
\hline $\mathrm{CHE}(\mathrm{U} / \mathrm{L})$ & $314.7 \pm 62.0$ & $378.7 \pm 67.5$ & $<0.0001$ & $315.1 \pm 63.9$ & $358.9 \pm 65.5$ & $<0.0001$ \\
\hline $\mathrm{TG}(\mathrm{mg} / \mathrm{dL})$ & $92.0 \pm 83.3$ & $157.7 \pm 108.3$ & $<0.0001$ & $89.6 \pm 50.4$ & $143.5 \pm 115.2$ & $<0.0001$ \\
\hline T-Chol (mg/dL) & $199.1 \pm 34.6$ & $208.4 \pm 34.0$ & $<0.0001$ & $202.1 \pm 35.6$ & $197.9 \pm 33.3$ & N.S. \\
\hline HDL-C (mg/dL) & $65.5 \pm 13.9$ & $53.8 \pm 10.7$ & $<0.0001$ & $67.9 \pm 15.3$ & $56.5 \pm 12.3$ & $<0.0001$ \\
\hline LDL-C (mg/dL) & $120.8 \pm 33.0$ & $138.7 \pm 30.5$ & $<0.0001$ & $121.4 \pm 30.7$ & $125.7 \pm 29.1$ & $<0.05$ \\
\hline Uric Acid (mg/dL) & $5.30 \pm 1.35$ & $6.13 \pm 1.32$ & $<0.0001$ & $5.27 \pm 1.32$ & $5.89 \pm 1.26$ & $<0.0001$ \\
\hline $\mathrm{FBG}(\mathrm{mg} / \mathrm{dL})$ & $108.0 \pm 27.9$ & $120.4 \pm 32.6$ & $<0.0001$ & $107.9 \pm 22.7$ & $120.7 \pm 26.9$ & $<0.0001$ \\
\hline HbA1c (\%) & $6.09 \pm 0.99$ & $6.49 \pm 1.08$ & $<0.0001$ & $6.14 \pm 0.82$ & $6.52 \pm 0.99$ & $<0.0001$ \\
\hline Iron $(\mu \mathrm{g} / \mathrm{dL})$ & $111.1 \pm 38.2$ & $117.1 \pm 37.8$ & N.S. & $112.8 \pm 38.3$ & $121.0 \pm 38.3$ & $<0.005$ \\
\hline Platelet count $\left(\times 10^{4} / \mu \mathrm{L}\right)$ & $21.4 \pm 4.9$ & $21.8 \pm 5.0$ & N.S. & $21.3 \pm 5.3$ & $21.0 \pm 4.9$ & N.S. \\
\hline FIB4-index & $1.39 \pm 0.6$ & $1.30 \pm 0.61$ & $<0.05$ & $1.67 \pm 0.82$ & $1.61 \pm 1.03$ & $<0.05$ \\
\hline NFS & $-1.32 \pm 1.04$ & $-1.31 \pm 1.07$ & N.S. & $-1.23 \pm 1.17$ & $-1.02 \pm 1.13$ & $<0.05$ \\
\hline M2BP $(\mu \mathrm{g} / \mathrm{mL})$ & $1.06 \pm 0.61$ & $1.85 \pm 1.34$ & $<0.0001$ & $1.12 \pm 0.95$ & $1.61 \pm 1.30$ & $<0.0001$ \\
\hline
\end{tabular}

Values represent mean $\pm \mathrm{SD} ;{ }^{*} p$ values correspond to the comparison between groups without and with fatty liver disease; Wilcoxon's test for continuous factors and Pearson's chi-square test for categorical factors were used;

\# Pearson's chi-square test between data at baseline and follow-up. N.S.; not significant. Abbreviations: BMI, body 
mass index; SBP, systolic blood pressure; AST, aspartate aminotransferase; ALT, alanine aminotransferase; GGT, gamma glutamyltransferase; T-Bil, total bilirubin; $\mathrm{CHE}$, choline esterase; TG, triglyceride; T-Chol, total cholesterol; HDL-C, high density lipoprotein cholesterol; LDL-C, low density lipoprotein cholesterol; FBG, fasting blood glucose; FIB4-index, Fibrosis-4 index; NFS, NAFLD fibrosis score; M2BP, Mac-2 binding protein.

\subsection{Serum M2BP Levels were Significantly Correlated with Liver Enzymes and Metabolic-Related Variables}

The results of Pearson's correlations analyses between serum M2BP levels and other liver and metabolic parameters at baseline are summarized in Table 2. Serum M2BP levels were significantly and positively correlated with BMI, SBP, AST, ALT, GGT, CHE, TG, T-Chol, LDL-C, uric acid, FBG, and $\mathrm{HbA} 1 \mathrm{c}$, whereas they were negatively correlated with T-Bil and HDL-C. These data suggested that serum M2BP levels were higher in subjects with metabolic disorder condition and/or liver dysfunction.

Table 2. Correlation between serum M2BP levels and various parameters at baseline.

\begin{tabular}{|c|c|c|}
\hline \multirow{2}{*}{ Variable } & \multicolumn{2}{|c|}{ M2BP } \\
\hline & $r$ & $p$ Value \\
\hline Age (years old) & 0.023 & N.S. \\
\hline $\operatorname{BMI}\left(\mathrm{kg} / \mathrm{m}^{2}\right)$ & 0.374 & $<0.0001$ \\
\hline Alcohol consumption (g/week) & 0.018 & N.S. \\
\hline $\mathrm{SBP}(\mathrm{mm} \mathrm{Hg})$ & 0.233 & $<0.0001$ \\
\hline AST (U/L) & 0.328 & $<0.0001$ \\
\hline $\operatorname{ALT}(\mathrm{U} / \mathrm{L})$ & 0.351 & $<0.0001$ \\
\hline GGT (U/L) & 0.279 & $<0.0001$ \\
\hline T-Bil (mg/dL) & -0.105 & $<0.01$ \\
\hline Albumin (mg/dL) & 0.085 & $<0.05$ \\
\hline Creatinine $(\mathrm{mg} / \mathrm{dL})$ & -0.045 & N.S. \\
\hline CHE (U/L) & 0.303 & $<0.0001$ \\
\hline $\mathrm{TG}(\mathrm{mg} / \mathrm{dL})$ & 0.326 & $<0.0001$ \\
\hline T-Chol (mg/dL) & 0.113 & $<0.005$ \\
\hline HDL-C (mg/dL) & -0.191 & $<0.0001$ \\
\hline LDL-C (mg/dL) & 0.132 & $<0.0005$ \\
\hline Uric acid (mg/dL) & 0.115 & $<0.005$ \\
\hline $\mathrm{FBG}(\mathrm{mg} / \mathrm{dL})$ & 0.099 & $<0.05$ \\
\hline $\mathrm{HbA} 1 \mathrm{c}(\%)$ & 0.119 & $<0.005$ \\
\hline Iron $(\mu \mathrm{g} / \mathrm{dL})$ & -0.002 & N.S. \\
\hline Platelet count $\left(\times 10^{4} / \mu \mathrm{L}\right)$ & 0.043 & N.S. \\
\hline FIB4-index & 0.061 & N.S. \\
\hline NFS & 0.058 & N.S. \\
\hline
\end{tabular}

Abbreviations are defined in the notes for Table 1.

\subsection{Among Metabolic Syndrome-Related Diseases, Fatty Liver Disease Was an Independent Determinant for} Serum M2BP Levels

We investigated the relationships between serum M2BP levels and metabolic syndrome-related diseases by comparing serum M2BP levels in each metabolic syndrome-related disease (obesity, hypertension, dyslipidemia, diabetes mellitus, and fatty liver) at baseline (Table 3A). Serum M2BP levels were higher in subjects with obesity, hypertension, dyslipidemia, diabetes mellitus, and fatty liver than in those without these diseases. We investigated which of these metabolic syndrome-related diseases were independent determinants for serum M2BP levels. Multiple logistic regression analyses indicated that obesity and fatty liver were independent determinants for serum M2BP levels (Table 3B). In male subjects, serum M2BP levels were higher in subjects with all metabolic syndrome-related diseases (Table S1A). In female subjects, serum M2BP levels were higher in subjects with obesity, hypertension, dyslipidemia, and fatty liver, but not with diabetes mellitus (Table S1B). Multiple logistic regression analyses indicated that obesity, hypertension, diabetes mellitus, and fatty liver were 
independent determinants for serum M2BP levels in male, and fatty liver was an only independent determinant for serum M2BP levels in female (Table S1C,D).

Table 3. Relationship between serum M2BP levels and metabolic risk factors. (A) Comparisons between serum M2BP levels and each metabolic syndrome-related disease at baseline. (B) Multiple logistic regression analysis of factors associated with serum M2BP levels at baseline.

\begin{tabular}{|c|c|c|c|c|}
\hline & (A) & & & \\
\hline Disease & Positive & Negative & \multicolumn{2}{|c|}{$p$ Value } \\
\hline Obesity & $1.88 \pm 1.38$ & $1.29 \pm 0.92$ & \multicolumn{2}{|c|}{$<0.0001$} \\
\hline Hypertension & $1.80 \pm 1.10$ & $1.41 \pm 1.20$ & \multicolumn{2}{|c|}{$<0.0001$} \\
\hline Dyslipidemia & $1.77 \pm 1.11$ & $1.40 \pm 1.20$ & \multicolumn{2}{|c|}{$<0.0001$} \\
\hline Diabetes mellitus & $1.62 \pm 1.30$ & $1.43 \pm 0.95$ & \multicolumn{2}{|c|}{$<0.05$} \\
\hline Fatty liver & $1.85 \pm 1.34$ & $1.06 \pm 0.61$ & \multicolumn{2}{|c|}{$<0.0001$} \\
\hline \multicolumn{5}{|c|}{ (B) } \\
\hline \multirow{2}{*}{ Factor } & \multirow{2}{*}{$t$ Value } & \multirow{2}{*}{$p$ Value } & \multicolumn{2}{|c|}{$95 \% \mathrm{CI}$} \\
\hline & & & Lower & Upper \\
\hline Obesity $(y=1, n=2)$ & 3 & $<0.005$ & 0.0496 & 0.237 \\
\hline Hypertension $(y=1, n=2)$ & 1.64 & N.S. & -0.0150 & 0.168 \\
\hline Dyslipidemia $(y=1, n=2)$ & 0.4 & N.S. & -0.0732 & 0.110 \\
\hline Diabetes mellitus $(y=1, n=2)$ & 0.95 & N.S. & -0.0444 & 0.127 \\
\hline Fatty liver $(y=1, n=2)$ & 5.83 & $<0.0001$ & 0.197 & 0.396 \\
\hline
\end{tabular}

Abbreviations are defined in the notes for Table 1 .

\subsection{Serum M2BP Levels Were Significantly Correlated with BMI in FL+ Subjects but Not in FL-Subjects}

Next, we investigated the relationships between serum M2BP levels and BMI in subjects with or without fatty liver disease (Figure 1). Serum M2BP levels were not correlated with BMI in FL- subjects, but were significantly correlated with BMI in FL+ subjects.

(A)

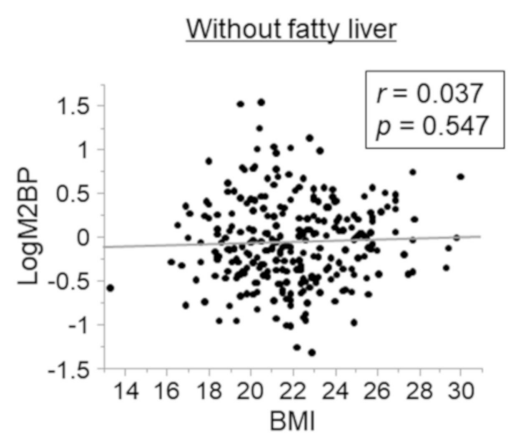

(B)

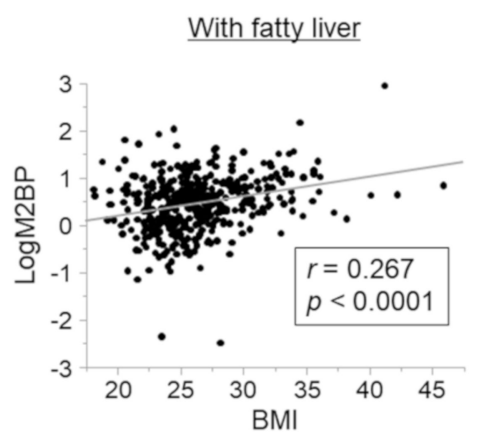

Figure 1. Relationships between serum M2BP levels and body mass index at baseline in subjects with or without fatty liver disease. (A) Relationships between serum M2BP levels and BMI in subjects without fatty liver. There were no significant relationships between serum M2BP levels and BMI in subjects without fatty liver. (B) Relationships between serum M2BP levels and BMI in subjects with fatty liver. There were significant positive relationships between serum M2BP levels and BMI in subjects with fatty liver.

\subsection{Relationships between Serum M2BP Levels at Baseline and Changes in Various Parameters}

We investigated the relationships between baseline serum M2BP levels and changes in various parameters (Table 4). Baseline M2BP levels were significantly and negatively correlated with changes in CHE and LDL-C, which are produced in the liver and represent hepatic synthetic capacity. Thus, higher serum M2BP levels would predict reduced hepatic synthetic capacity. We also observed that baseline M2BP levels were significantly and negatively correlated with changes in platelet count, 
and significantly and positively correlated with changes in FIB4-index and NFS. This result indicated that higher serum M2BP level could predict liver fibrosis progression.

Table 4. Correlations between baseline serum M2BP levels and changes in various parameters.

\begin{tabular}{ccc}
\hline & \multicolumn{2}{c}{ M2BP } \\
\hline Variable & $r$ & $p$ Value \\
\hline$\Delta$ BMI $\left(\mathrm{kg} / \mathrm{m}^{2}\right)$ & -0.094 & $<0.05$ \\
$\Delta$ alcohol consumption $(\mathrm{g} / \mathrm{week})$ & 0.031 & N.S. \\
$\Delta$ SBP $(\mathrm{mm} \mathrm{Hg})$ & -0.087 & $<0.05$ \\
$\Delta$ AST $(\mathrm{U} / \mathrm{L})$ & 0.012 & N.S. \\
$\Delta$ ALT $(\mathrm{U} / \mathrm{L})$ & -0.069 & N.S. \\
$\Delta$ GGT $(\mathrm{U} / \mathrm{L})$ & -0.032 & N.S. \\
$\Delta$ T-Bil $(\mathrm{mg} / \mathrm{dL})$ & 0.111 & N.S. \\
$\Delta$ Albumin $(\mathrm{mg} / \mathrm{dL})$ & -0.042 & N.S. \\
$\Delta$ Creatinine $(\mathrm{mg} / \mathrm{dL})$ & 0.013 & N.S. \\
$\Delta$ CHE $(\mathrm{U} / \mathrm{L})$ & -0.196 & $<0.0001$ \\
$\Delta$ TG $(\mathrm{mg} / \mathrm{dL})$ & -0.157 & N.S. \\
$\Delta$ T-Chol $(\mathrm{mg} / \mathrm{dL})$ & -0.187 & $<0.05$ \\
$\Delta$ HDL-C $(\mathrm{mg} / \mathrm{dL})$ & 0.001 & N.S. \\
$\Delta$ LDL-C $(\mathrm{mg} / \mathrm{dL})$ & -0.152 & $<0.001$ \\
$\Delta$ Uric acid $(\mathrm{mg} / \mathrm{dL})$ & -0.080 & N.S. \\
$\Delta$ FBG $(\mathrm{mg} / \mathrm{dL})$ & 0.039 & N.S. \\
$\Delta$ HbA1c $(\%)$ & -0.0056 & N.S. \\
$\Delta$ Iron $(\mu \mathrm{d} / \mathrm{dL})$ & 0 & N.S. \\
$\Delta$ Platelet count $\left(\times 10^{4} / \mu \mathrm{L}\right)$ & -0.19 & $<0.05$ \\
$\Delta$ FIB4-index & 0.2 & $<0.0001$ \\
$\Delta$ NFS & 0.18 & $<0.0001$ \\
\hline
\end{tabular}

Abbreviations are defined in the notes for Table $1 ; \Delta$, change in each parameter at 7-year follow-up.

\subsection{Serum M2BP Levels Could Serve as a Predictive Biomarker for Liver Fibrosis Progression}

Serum M2BP levels were not significantly correlated with platelet count, FIB4-index, or NFS at baseline (Table 2). However, the four parameters of M2BP, platelet count, FIB4-index, and NFS are useful liver fibrosis markers for fatty liver disease [12,36-38]. We previously measured serum M2BP levels in 512 biopsy-proven NAFLD patients, and set a cut-off value $(1.80 \mu \mathrm{g} / \mathrm{mL})$ for the diagnosis of NASH from NAFLD [28]. In the present study, we compared the ability to predict changes in FIB4-index using the baseline values of FIB4-index, NFS, and serum M2BP levels (Table 5). Both FIB-4 index and NFS have two cut-off points (FIB4-index: low cut-off point, 1.30; high cut-off point, 2.67, NFS: low cut-off point, -1.455; high cut-off point, 0.676) [12,35]. We stratified study subjects based on low FIB4-index cut-off point (1.30), low NFS cut-off point (-1.455), or serum M2BP level $(1.80 \mu \mathrm{g} / \mathrm{mL})$, and investigated the relationships between these parameters and changes in the FIB4-index. The baseline FIB4-index and NFS were not correlated with changes in the FIB4-index. By contrast, baseline serum M2BP levels were significantly correlated with changes in the FIB4-index, and this correlation became stronger in subjects with higher baseline serum M2BP levels. These results indicated that subjects with higher serum M2BP levels at baseline would undergo liver fibrosis progression.

Table 5. Correlations between FIB4-index, NFS, or serum M2BP levels at baseline and changes in FIB4-index divided by cut-off values of FIB4-index, NFS, or serum M2BP levels.

\begin{tabular}{|c|c|c|c|c|c|c|c|c|c|c|c|c|}
\hline & \multicolumn{4}{|c|}{ FIB4-Index } & \multicolumn{4}{|c|}{ NFS } & \multicolumn{4}{|c|}{ М2BP } \\
\hline & \multicolumn{2}{|c|}{$\begin{array}{c}<1.30 \\
(n=424)\end{array}$} & \multicolumn{2}{|c|}{$\begin{array}{c}1.30< \\
(n=291)\end{array}$} & \multicolumn{2}{|c|}{$\begin{array}{l}<-1.455 \\
(n=303)\end{array}$} & \multicolumn{2}{|c|}{$\begin{array}{l}-1.455< \\
(n=379)\end{array}$} & \multicolumn{2}{|c|}{$\begin{array}{c}<1.80 \mu \mathrm{g} / \mathrm{Ml} \\
\quad(n=510)\end{array}$} & \multicolumn{2}{|c|}{$\begin{array}{c}1.80 \mu \mathrm{g} / \mathrm{mL}< \\
(n=205)\end{array}$} \\
\hline & $r$ & $p$ Value & $R$ & $p$ Value & $r$ & $p$ Value & $r$ & $p$ Value & $r$ & $p$ Value & $r$ & $p$ Value \\
\hline$\Delta$ FIB4-index & 0.048 & N.S. & 0.096 & N.S. & 0.099 & N.S. & 0.16 & $<0.005$ & 0.111 & $<0.05$ & 0.309 & $<0.0001$ \\
\hline
\end{tabular}

Abbreviations are defined in the notes for Table 1. 
Decreased blood platelet count, FIB4-index, and NFS are useful clinical biomarkers for liver fibrosis. We performed multivariate analysis to identify baseline parameters that predicted changes in platelet count (Table 6A), FIB4-index (Table 6B), and NFS (Table S2). We found that BMI and serum M2BP levels were significant negative determinants for platelet count changes, whereas serum TG and iron levels were significant positive determinants for platelet count changes. We also found that serum M2BP level was a significant positive determinant for FIB4-index changes, whereas serum TG was a significant negative determinant for FIB4-index changes. Serum M2BP level, age, ALT, albumin, $\mathrm{TG}$, and $\mathrm{HbA} 1 \mathrm{c}$ were significant positive determinants for NFS changes, whereas serum iron was a significant negative determinant for NFS changes (Table S2).

Table 6. M2BP could be a predictive biomarker for liver fibrosis progression. (A) Multivariate analysis of predicted changes in platelet count using baseline various variables. (B) Multivariate analysis of predicted changes in FIB4-index using baseline various variables.

\begin{tabular}{|c|c|c|c|c|}
\hline \multicolumn{5}{|c|}{ (A) } \\
\hline \multirow{2}{*}{ Variable } & \multirow{2}{*}{$t$ Value } & \multirow{2}{*}{$p$ Value } & \multicolumn{2}{|c|}{$95 \% \mathrm{CI}$} \\
\hline & & & Lower & Upper \\
\hline Gender $(F)$ & 0.93 & N.S. & -0.232 & 0.645 \\
\hline Age & -1.48 & N.S. & -0.06942 & 0.00980 \\
\hline BMI $\left(\mathrm{kg} / \mathrm{m}^{2}\right)$ & -2.01 & $<0.05$ & -0.170 & -0.0018 \\
\hline Alcohol consumption (g/week) & -1.24 & N.S. & -0.0042 & 0.000948 \\
\hline $\mathrm{SBP}(\mathrm{mm} \mathrm{Hg})$ & -0.34 & N.S. & -0.0227 & 0.0160 \\
\hline $\operatorname{ALT}(\mathrm{U} / \mathrm{L})$ & -0.71 & N.S. & -0.0195 & 0.00918 \\
\hline GGT (U/L) & -0.72 & N.S. & -0.00553 & 0.00258 \\
\hline T-Bil (mg/dL) & 0.57 & N.S. & -0.703 & 1.28 \\
\hline Albumin (mg/dL) & -0.99 & N.S. & -1.83 & 0.605 \\
\hline Creatinine (mg/dL) & 1.5 & N.S. & -0.546 & 4.05 \\
\hline $\mathrm{CHE}(\mathrm{U} / \mathrm{L})$ & -0.43 & N.S. & -0.00569 & 0.00365 \\
\hline $\mathrm{TG}(\mathrm{mg} / \mathrm{dL})$ & 2.94 & $<0.005$ & 0.00151 & 0.00756 \\
\hline T-Chol (mg/dL) & -1.61 & N.S. & -0.0155 & 0.00154 \\
\hline Uric acid (mg/dL) & -0.56 & N.S. & -0.322 & 0.179 \\
\hline Iron $(\mu \mathrm{g} / \mathrm{dL})$ & 2.39 & $<0.05$ & 0.00171 & 0.0176 \\
\hline $\mathrm{FBG}(\mathrm{mg} / \mathrm{dL})$ & 1.86 & N.S. & -0.00091 & 0.0327 \\
\hline $\mathrm{HbA1c}(\%)$ & -0.93 & N.S. & -0.751 & 0.269 \\
\hline \multirow[t]{2}{*}{ M2BP $(\mu \mathrm{g} / \mathrm{mL})$} & -2.93 & $<0.005$ & -0.628 & -0.124 \\
\hline & \multicolumn{2}{|c|}{ (B) } & & \\
\hline \multirow{2}{*}{ Variable } & \multirow{2}{*}{$t$ Value } & \multirow{2}{*}{$p$ Value } & \multicolumn{2}{|c|}{$95 \%$ CI } \\
\hline & & & Lower & Upper \\
\hline Gender $(F)$ & -1.75 & N.S. & -0.151 & 0.00889 \\
\hline Age & 1.98 & N.S. & $5.51 \times 10^{-5}$ & 0.0145 \\
\hline BMI $\left(\mathrm{kg} / \mathrm{m}^{2}\right)$ & 0.75 & N.S. & -0.00954 & 0.0213 \\
\hline Alcohol consumption (g/week) & 0.62 & N.S. & -0.00032 & 0.000619 \\
\hline SBP $(\mathrm{mm} \mathrm{Hg})$ & -0.86 & N.S. & -0.00509 & 0.00200 \\
\hline $\operatorname{ALT}(\mathrm{U} / \mathrm{L})$ & -1.43 & N.S. & -0.00454 & 0.00071 \\
\hline GGT (U/L) & 1.19 & N.S. & -0.00029 & 0.00119 \\
\hline T-Bil (mg/dL) & 1.12 & N.S. & -0.0776 & 0.286 \\
\hline Albumin (mg/dL) & -0.92 & N.S. & -0.326 & 0.119 \\
\hline Creatinine (mg/dL) & -1.35 & N.S. & -0.709 & 0.132 \\
\hline $\mathrm{CHE}(\mathrm{U} / \mathrm{L})$ & -1.23 & N.S. & -0.00139 & 0.00032 \\
\hline $\mathrm{TG}(\mathrm{mg} / \mathrm{dL})$ & -3.46 & $<0.001$ & -0.00153 & -0.00042 \\
\hline T-Chol (mg/dL) & -0.62 & N.S. & -0.00205 & 0.00107 \\
\hline Uric acid (mg/dL) & 0.97 & N.S. & -0.0232 & 0.0685 \\
\hline Iron $(\mu \mathrm{g} / \mathrm{dL})$ & -1.07 & N.S. & -0.00224 & 0.000661 \\
\hline FBG (mg/dL) & 0.25 & N.S. & -0.00269 & 0.00346 \\
\hline $\mathrm{HbA1c}(\%)$ & 0.03 & N.S. & -0.0921 & 0.0944 \\
\hline M2BP $(\mu \mathrm{g} / \mathrm{mL})$ & 11.89 & $<0.0001$ & 0.233 & 0.325 \\
\hline
\end{tabular}

Abbreviations are defined in the notes for Table $1 ; \mathrm{CI}$, confidence interval. 


\subsection{Relationships between Changes in M2BP Levels and Other Parameters}

Next, we investigated the relationships between changes in serum M2BP levels and other parameters (Table 7A). Changes in serum M2BP levels were significantly and positively correlated with changes in BMI, alcohol consumption, SBP, AST, ALT, GGT, CHE, TG, T-Chol, LDL-C, FBG, and $\mathrm{HbA} 1 \mathrm{c}$, whereas changes in HDL-C were significantly and negatively correlated with changes in serum M2BP levels. These results indicated that serum M2BP levels would increase with metabolic syndrome progression and liver dysfunction.

Table 7. Changes in serum M2BP levels were correlated with changes in parameters related to metabolic risk. (A) Correlation coefficients for changes in M2BP levels and changes in various variables. (B) Multivariate analysis of predicted changes in serum M2BP levels using changes in various variables.

\begin{tabular}{|c|c|c|c|c|}
\hline \multicolumn{5}{|c|}{ (A) } \\
\hline \multirow{2}{*}{\multicolumn{2}{|c|}{ Variable }} & \multicolumn{3}{|c|}{$\Delta \mathrm{M} 2 \mathrm{BP}$} \\
\hline & & & $r$ & $p$ Value \\
\hline \multicolumn{2}{|l|}{$\Delta \mathrm{BMI}\left(\mathrm{kg} / \mathrm{m}^{2}\right)$} & \multicolumn{2}{|r|}{0.136} & $<0.001$ \\
\hline \multicolumn{2}{|l|}{$\Delta$ alcohol consumption (g/week) } & \multicolumn{2}{|r|}{0.082} & $<0.05$ \\
\hline \multicolumn{2}{|l|}{$\Delta \mathrm{SBP}(\mathrm{mm} \mathrm{Hg})$} & \multicolumn{2}{|r|}{0.114} & $<0.005$ \\
\hline$\Delta \mathrm{AST}(\mathrm{U} / \mathrm{L})$ & & \multicolumn{2}{|r|}{0.118} & $<0.005$ \\
\hline$\triangle \mathrm{ALT}(\mathrm{U} / \mathrm{L})$ & & \multicolumn{2}{|r|}{0.089} & $<0.05$ \\
\hline$\Delta \mathrm{GGT}(\mathrm{U} / \mathrm{L})$ & & \multicolumn{2}{|r|}{0.153} & $<0.0005$ \\
\hline$\Delta$ Albumin $(\mathrm{mg} / \mathrm{dL})$ & & \multicolumn{2}{|r|}{0.01} & N.S. \\
\hline$\Delta \mathrm{CHE}(\mathrm{U} / \mathrm{L})$ & & \multicolumn{2}{|r|}{0.183} & $<0.001$ \\
\hline$\Delta \mathrm{TG}(\mathrm{mg} / \mathrm{dL})$ & & \multicolumn{2}{|r|}{0.1} & $<0.05$ \\
\hline$\Delta \mathrm{T}-\mathrm{Chol}(\mathrm{mg} / \mathrm{dL})$ & & \multicolumn{2}{|r|}{0.133} & $<0.0005$ \\
\hline$\Delta \mathrm{HDL}-\mathrm{C}(\mathrm{mg} / \mathrm{dL})$ & & \multicolumn{2}{|r|}{-0.11} & $<0.01$ \\
\hline$\Delta \mathrm{LDL}-\mathrm{C}(\mathrm{mg} / \mathrm{dL})$ & & \multicolumn{2}{|r|}{0.135} & $<0.001$ \\
\hline$\Delta$ Uric acid $(\mathrm{mg} / \mathrm{dL})$ & & \multicolumn{2}{|r|}{-0.016} & N.S. \\
\hline$\Delta$ Creatinine $(\mathrm{mg} / \mathrm{dL})$ & & \multicolumn{2}{|r|}{-0.014} & N.S. \\
\hline$\Delta \mathrm{FBG}(\mathrm{mg} / \mathrm{dL})$ & & \multicolumn{2}{|r|}{0.14} & $<0.001$ \\
\hline$\Delta \mathrm{HbA} 1 \mathrm{c}(\%)$ & & \multicolumn{2}{|r|}{0.177} & $<0.001$ \\
\hline$\Delta \operatorname{Iron}(\mu \mathrm{g} / \mathrm{dL})$ & & \multicolumn{2}{|r|}{-0.046} & N.S. \\
\hline$\Delta$ Platelet count $\left(\times 10^{4} / \mu \mathrm{L}\right)$ & & \multicolumn{2}{|r|}{-0.046} & N.S. \\
\hline$\Delta$ FIB4-index & & \multicolumn{2}{|r|}{-0.051} & N.S. \\
\hline$\triangle \mathrm{NFS}$ & & \multicolumn{2}{|r|}{-0.039} & N.S. \\
\hline & (B) & & & \\
\hline & & $n$ Value & $95^{\circ}$ & \\
\hline Variable & $t$ Value & $p$ value & Lower & Upper \\
\hline$\Delta \mathrm{BMI}\left(\mathrm{kg} / \mathrm{m}^{2}\right)$ & 1.04 & N.S. & -0.0290 & 0.0942 \\
\hline$\Delta$ alcohol consumption (g/week) & 0.47 & N.S. & $-8.89 \times 10^{-4}$ & 0.00146 \\
\hline$\Delta \mathrm{SBP}(\mathrm{mm} \mathrm{Hg})$ & 2.32 & $<0.05$ & 0.00105 & 0.0126 \\
\hline$\Delta \mathrm{ALT}(\mathrm{U} / \mathrm{L})$ & -0.43 & N.S. & -0.005323 & 0.00342 \\
\hline$\Delta \mathrm{GGT}(\mathrm{U} / \mathrm{L})$ & 2.69 & $<0.01$ & 0.000603 & 0.00386 \\
\hline$\Delta$ Albumin $(\mathrm{mg} / \mathrm{dL})$ & 0.47 & N.S. & -0.345 & 0.562 \\
\hline$\Delta \mathrm{CHE}(\mathrm{U} / \mathrm{L})$ & 2.27 & $<0.05$ & 0.000371 & 0.00516 \\
\hline$\Delta \mathrm{TG}(\mathrm{mg} / \mathrm{dL})$ & 0.18 & N.S. & -0.00101 & 0.00122 \\
\hline$\Delta \mathrm{T}-\mathrm{Chol}(\mathrm{mg} / \mathrm{dL})$ & 1.31 & N.S. & -0.000987 & 0.00494 \\
\hline$\Delta$ Uric acid $(\mathrm{mg} / \mathrm{dL})$ & -1.27 & N.S. & -0.147 & 0.0315 \\
\hline$\Delta$ Creatinine $(\mathrm{mg} / \mathrm{dL})$ & 1.36 & N.S. & -0.132 & 0.725 \\
\hline$\Delta \mathrm{FBG}(\mathrm{mg} / \mathrm{dL})$ & -0.07 & N.S. & -0.00489 & 0.00453 \\
\hline$\Delta \mathrm{HbA} 1 \mathrm{c}(\%)$ & 2.37 & $<0.05$ & 0.0314 & 0.335 \\
\hline$\Delta \operatorname{Iron}(\mu \mathrm{g} / \mathrm{dL})$ & -1.94 & N.S. & -0.00382 & $2.63 \times 10^{-5}$ \\
\hline$\Delta$ Platelet count $\left(\times 10^{4} / \mu \mathrm{L}\right)$ & 0.78 & N.S. & -0.0156 & 0.0360 \\
\hline
\end{tabular}

Abbreviations are defined in the notes for Table 1. 


\subsection{Changes in GGT and CHE Were Independent Determinants for Changes in M2BP Levels}

Finally, we performed multivariate analyses to investigate which parameter changes could predict changes in serum M2BP levels (Table 7B). The results indicated that changes in SBP, GGT, CHE, and $\mathrm{HbA1c}$ were significantly and positively correlated with changes in serum M2BP levels. In male subjects, changes in SBP, ALT, and GGT were significantly and positively correlated with changes in serum M2BP levels (Table S3A). In female subjects, CHE was positively and ALT was negatively correlated with changes in serum M2BP levels (Table S3B). These effects of gender differences on changes in M2BP would need further investigation.

\section{Discussion}

We recently reported that M2BP was a useful biomarker that discriminated NAFLD from NASH $[18,21,28,36]$. Serum M2BP level is a useful predictive biomarker for NAFLD fibrosis progression, and M2BP levels increase during the progression of fibrosis. To investigate whether serum M2BP level can predict the development of liver fibrosis, we conducted a 7-year longitudinal study. There are four main results of our study: (1) serum M2BP levels at baseline were significantly correlated with changes in platelet count, FIB-4 index, and NFS, which are clinically used as liver fibrosis markers; (2) serum M2BP levels were most significantly correlated with fatty liver among the metabolic syndrome-related diseases; (3) serum M2BP levels were significantly correlated with BMI in subjects with fatty liver, but not in subjects without fatty liver; (4) changes in SBP, GGT, CHE, and HbA1c were independent determinants for changes in M2BP levels. These combined results indicated that serum M2BP level was closely associated with fatty liver disease and would serve as a predictive biomarker for liver fibrosis progression in patients with fatty liver disease.

Serum M2BP levels were not correlated with platelet count, FIB-4 index, and NFS at baseline. Our previous study demonstrated serum M2BP levels significantly correlated with FIB4-index and NFS $[21,28]$. In the present study, our study subjects were those who had received health check-ups and there would be few subjects with advanced liver fibrosis. The frequency of subjects whose value of FIB4-index was over 2.67 was $14.3 \%$ (73 among 512 NAFLD patients) in our previous study, while it was 3.1\% (22 among 715 subjects) in this study at baseline. This difference in the study subjects between our previous study and present study would have any significance on these relationships between serum M2BP levels and liver fibrosis biomarkers. Platelet count decreases with liver fibrosis progression, and platelet count are often used as a clinical liver fibrosis marker. FIB4-index and NFS are scoring systems developed for the assessment of liver fibrosis progression [12,34,35]. Our longitudinal study demonstrated that baseline serum M2BP levels were significant determinants for platelet count decrease, FIB4-index increase, and NFS increase. Our present results indicated that baseline serum M2BP levels could predict liver fibrosis progression.

Recent studies demonstrated that a screening test using FIB4-index improved the management of patients with fatty liver $[39,40]$. Based on the results of our present study, we developed a two-step noninvasive protocol for the management of patients with metabolic fatty liver (Figure S1). Step 1 was a calculation of the FIB4-index. Subjects with FIB4-index $<1.30$ (424 subjects in this study) were considered as being at low risk of advanced liver fibrosis; they remained in clinical observation with re-assessment of advanced liver fibrosis risk after several years. Subjects with FIB-4 index $>2.67$ (22 subjects) were considered as high risk of advanced liver fibrosis, and were recommended for liver biopsy and detailed management by hepatologists. Step 2 was a measurement of serum M2BP levels. For the diagnosis of NASH from NAFLD, we set the cut-off value of serum M2BP as $1.80 \mu \mathrm{g} / \mathrm{mL}$ in our previous study [28]. Subjects with intermediate FIB4-index of 1.30-2.67 (269 subjects) received a test to measure serum M2BP levels; those with serum M2BP levels $<1.80 \mu \mathrm{g} / \mathrm{mL}$ (179 subjects) remained under clinical observation, whereas those with serum M2BP levels $>1.80 \mu \mathrm{g} / \mathrm{mL}$ (90 subjects) were recommended for detailed management by hepatologists. According to our protocol, about one-third of subjects with intermediate FIB4-index were recommended for detailed management. 
Our previous study used various mouse liver disease models to analyze serum M2BP levels in liver fibrosis progression and liver inflammation [41]. M2BP belongs to the Scavenger receptor cysteine-rich domain (SRCR) superfamily of proteins involved in immune defense and regulation [42]. M2BP is expressed in many tissues and various types of cell including macrophages, hepatocytes, and hepatic stellate cells. Its expression in mouse macrophages can be upregulated by adherence and inflammatory cytokines (tumor necrosis factor- $\alpha$ (TNF- $\alpha$ ) and interferon- $\gamma$ (IFN- $\gamma$ )) [43]. In chronic and acute liver injury mouse models, gene expression of M2BP significantly increased in liver [41]. Increased serum M2BP levels in liver diseases would mainly be produced from liver. Considering these findings, we believe that M2BP is associated with immunoreaction of liver fibrosis [23,25,27,44]. Although some differences in the molecular structures of human and mouse M2BP have been reported, M2BP-deficient mice exhibited a higher death rate after intraperitoneal lipopolysaccharide administration than wild-type mice [43]. This result indicated that inflammation-induced M2BP increase would have anti-inflammatory effects as a defensive substrate. In our study, subjects with higher M2BP levels would have enhanced liver inflammation, but the anti-inflammatory effects of M2BP would be not sufficient to suppress inflammation, which led to further liver fibrosis progression during the 7-year study. In addition, our study demonstrated fatty liver induced by aggravated nutritional status would have significant roles in changes in serum M2BP levels. Correcting nutritional status in fatty liver patients should reduce serum M2BP levels and lead to the improvement of fatty liver disease.

Our study has some limitations. First, the major limitation of these types of study is that only healthy subjects during 7-years tended to be analyzed. Second, we did not perform liver biopsy in our study subjects and could not directly examine whether serum M2BP levels predicted liver fibrosis progression. Our study subjects received health examinations, and liver biopsy was not feasible in this study for ethical reasons. Third, a follow-up period of 7 years might not be sufficient to investigate a possible role of M2BP in the progression of liver fibrosis. Forth, this is an observation study that cannot investigate the effects of interventional therapies on changes in serum M2BP levels. In future studies, we will resolve these limitations by including biopsy-proven liver disease patients. Fifth, we could not obtain data about menopause, we did not analyze the effects of menopause in female subjects. Our demonstrated data were sometimes different between male and female subjects. Female hormone would have a significance on these distinctions.

\section{Conclusions}

In conclusion, this is the first prospective observational study that demonstrates relationships between serum M2BP levels and other metabolic parameters. We found that serum M2BP levels increased in various metabolic syndrome-related diseases, especially in fatty liver disease. In addition, serum M2BP levels significantly associated with metabolic parameters, and nutritional status resulted in significant changes in serum M2BP levels. In addition, we found that serum M2BP levels at baseline could be used to predict changes in FIB4-index and NFS, which are useful liver fibrosis scoring systems. Our results indicate the importance of measuring serum M2BP levels in the management of patients with fatty liver disease.

Supplementary Materials: The following are available online at http:/www.mdpi.com/2072-6643/12/6/1770/s1, Figure S1: Proposed management strategy algorithm for nonalcoholic fatty liver disease, Table S1: Relationship between serum M2BP levels and metabolic risk factors in male and female, Table S2: Multivariate analysis of predicted changes in NFS using baseline various variables, Table S3: Multivariate analysis of predicted changes in serum M2BP levels using changes in various variables by gender.

Author Contributions: Conceptualization, Y.K. and E.M.; methodology, Y.K. and K.M.; investigation, M.N., T.A., Y.N., and M.Y.; writing—original draft preparation, Y.K.; writing—review and editing, M.K., S.T., Y.S., T.T. and E.M.; supervision, E.M.; funding acquisition, Y.K. All authors have read and agreed to the published version of the manuscript.

Funding: This study was supported by JSPS KAKENHI grants (Grant Number 19H03562, 20K08383) from the Japan Society for the Promotion of Science, and a grant from the Program for Basic and Clinical Research on Hepatitis from Japan Agency for Medical Research and development (AMED) (Grant Number JP19fk0210031). 
Acknowledgments: The authors thank Eriko Matsuno, Kohei Kita, Saki Itoyama, and Nami Ito for their excellent technical assistance.

Conflicts of Interest: All authors declare that they have no conflicts of interest.

\section{References}

1. Ford, E.S.; Giles, W.H.; Dietz, W.H. Prevalence of the metabolic syndrome among us adults: Findings from the third national health and nutrition examination survey. JAMA 2002, 287, 356-359. [CrossRef] [PubMed]

2. Shirabe, K.; Takeishi, K.; Taketomi, A.; Uchiyama, H.; Kayashima, H.; Maehara, Y. Improvement of Long-Term Outcomes in Hepatitis C Virus Antibody-Positive Patients with Hepatocellular Carcinoma after Hepatectomy in the Modern Era. World J. Surg. 2011, 35, 1072-1084. [CrossRef] [PubMed]

3. Mazzaferro, V.; Regalia, E.; Doci, R.; Andreola, S.; Pulvirenti, A.; Bozzetti, F.; Montalto, F.; Ammatuna, M.; Morabito, A.; Gennari, L. Liver Transplantation for the Treatment of Small Hepatocellular Carcinomas in Patients with Cirrhosis. N. Engl. J. Med. 1996, 334, 693-700. [CrossRef]

4. Bravo, A.A.; Sheth, S.G.; Chopra, S. Liver Biopsy. N. Engl. J. Med. 2001, 344, 495-500. [CrossRef] [PubMed]

5. Gebo, K.A.; Herlong, H.F.; Torbenson, M.S.; Jenckes, M.W.; Chander, G.; Ghanem, K.G.; El-Kamary, S.S.; Sulkowski, M.; Bass, E.B. Role of liver biopsy in management of chronic hepatitis C: A systematic review. Hepatology 2002, 36, s161-s172. [CrossRef]

6. Piccinino, F.; Sagnelli, E.; Pasquale, G.; Giusti, G. Complications following percutaneous liver biopsy. A multicentre retrospective study on 68,276 biopsies. J. Hepatol. 1986, 2, 165-173. [CrossRef]

7. Ratziu, V.; Charlotte, F.; Heurtier, A.; Gombert, S.; Giral, P.; Bruckert, E.; Grimaldi, A.; Capron, F.; Poynard, T. Sampling variability of liver biopsy in nonalcoholic fatty liver disease. Gastroenterology 2005, 128, 1898-1906. [CrossRef]

8. Angulo, P.; Kleiner, D.; Dam-Larsen, S.; Adams, L.A.; Björnsson, E.S.; Charatcharoenwitthaya, P.; Mills, P.R.; Keach, J.C.; Lafferty, H.D.; Stahler, A.; et al. Liver Fibrosis, but No Other Histologic Features, Is Associated with Long-term Outcomes of Patients with Nonalcoholic Fatty Liver Disease. Gastroenterology 2015, 149, 389-397.e10. [CrossRef]

9. Yoneda, M.; Fujita, K.; Inamori, M.; Nakajima, A.; Tamano, M.; Hiraishi, H. Transient elastography in patients with non-alcoholic fatty liver disease (NAFLD). Gut 2007, 56, 1330-1331. [CrossRef]

10. Yoneda, M.; Suzuki, K.; Kato, S.; Fujita, K.; Nozaki, Y.; Hosono, K.; Saito, S.; Nakajima, A. Nonalcoholic Fatty Liver Disease: US-based Acoustic Radiation Force Impulse Elastography. Radiology 2010, 256, 640-647. [CrossRef]

11. Castera, L.; Forns, X.; Alberti, A. Non-invasive evaluation of liver fibrosis using transient elastography. J. Hepatol. 2008, 48, 835-847. [CrossRef] [PubMed]

12. Angulo, P.; Hui, J.M.; Marchesini, G.; Bugianesi, E.; George, J.; Farrell, G.C.; Enders, F.; Saksena, S.; Burt, A.; Bida, J.P.; et al. The NAFLD fibrosis score: A noninvasive system that identifies liver fibrosis in patients with NAFLD. Hepatology 2007, 45, 846-854. [CrossRef]

13. Dixon, J.; Bhathal, P.S.; O’Brien, P.E. Nonalcoholic fatty liver disease: Predictors of nonalcoholic steatohepatitis and liver fibrosis in the severely obese. Gastroenterology 2001, 121, 91-100. [CrossRef] [PubMed]

14. Harrison, S.A.; Oliver, D.; Arnold, H.L.; Gogia, S.; Neuschwander-Tetri, B. A Development and validation of a simple NAFLD clinical scoring system for identifying patients without advanced disease. Gut 2008, 57, 1441-1447. [CrossRef] [PubMed]

15. Sumida, Y.; Japan Study Group of Nonalcoholic Fatty Liver Disease (JSG-NAFLD); Yoneda, M.; Hyogo, H.; Yamaguchi, K.; Ono, M.; Fujii, H.; Eguchi, Y.; Suzuki, Y.; Imai, S.; et al. A simple clinical scoring system using ferritin, fasting insulin, and type IV collagen 7S for predicting steatohepatitis in nonalcoholic fatty liver disease. J. Gastroenterol. 2010, 46, 257-268. [CrossRef] [PubMed]

16. Grassadonia, A.; Tinari, N.; Iurisci, I.; Piccolo, E.; Cumashi, A.; Innominato, P.; D’Egidio, M.; Natoli, C.; Piantelli, M.; Iacobelli, S. 90K (Mac-2 BP) and galectins in tumor progression and metastasis. Glycoconj. J. 2002, 19, 551-556. [CrossRef]

17. Przybyło, M.; Martuszewska, D.; Pocheć, E.; Hoja-Łukowicz, D.; Litynska, A. Identification of proteins bearing $\beta 1-6$ branched N-glycans in human melanoma cell lines from different progression stages by tandem mass spectrometry analysis. Biochim. Biophys. Acta (BBA)-Gen. Subj. 2007, 1770, 1427-1435. [CrossRef] 
18. Kamada, Y.; Fujii, H.; Fujii, H.; Sawai, Y.; Doi, Y.; Uozumi, N.; Mizutani, K.; Akita, M.; Sato, M.; Kida, S.; et al. Serum Mac-2 binding protein levels as a novel diagnostic biomarker for prediction of disease severity and nonalcoholic steatohepatitis. Proteom. Clin. Appl. 2013, 7, 648-656. [CrossRef]

19. Artini, M.; Natoli, C.; Tinari, N.; Costanzo, A.; Marinelli, R.; Balsano, C.; Porcari, P.; Angelucci, D.; D’Egidio, M.; Levrero, M.; et al. Elevated serum levels of 90K/MAC-2 BP predict unresponsiveness to $\alpha$-interferon therapy in chronic HCV hepatitis patients. J. Hepatol. 1996, 25, 212-217. [CrossRef]

20. Cheung, K.J.; Tilleman, K.; Deforce, D.; Colle, I.; Van Vlierberghe, H. The HCV serum proteome: A search for fibrosis protein markers. J. Viral Hepat. 2009, 16, 418-429. [CrossRef]

21. Kamada, Y.; Ono, M.; Hyogo, H.; Fujii, H.; Sumida, Y.; Mori, K.; Tanaka, S.; Yamada, M.; Akita, M.; Mizutani, K.; et al. A novel noninvasive diagnostic method for nonalcoholic steatohepatitis using two glycobiomarkers. Hepatology 2015, 62, 1433-1443. [CrossRef] [PubMed]

22. Kuno, A.; Ikehara, Y.; Tanaka, Y.; Ito, K.; Matsuda, A.; Sekiya, S.; Hige, S.; Sakamoto, M.; Kage, M.; Mizokami, M.; et al. A serum "sweet-doughnut" protein facilitates fibrosis evaluation and therapy assessment in patients with viral hepatitis. Sci. Rep. 2013, 3, 1065. [CrossRef] [PubMed]

23. Abe, M.; Miyake, T.; Kuno, A.; Imai, Y.; Sawai, Y.; Hino, K.; Hara, Y.; Hige, S.; Sakamoto, M.; Yamada, G.; et al. Association between Wisteria floribunda agglutinin-positive Mac-2 binding protein and the fibrosis stage of non-alcoholic fatty liver disease. J. Gastroenterol. 2014, 50, 776-784. [CrossRef] [PubMed]

24. Ito, K.; Murotani, K.; Nakade, Y.; Inoue, T.; Nakao, H.; Sumida, Y.; Kamada, Y.; Yoneda, M. Serum Wisteria floribunda agglutinin-positive Mac-2-binding protein levels and liver fibrosis: A meta-analysis. J. Gastroenterol. Hepatol. 2017, 32, 1922-1930. [CrossRef]

25. Zou, X.; Zhu, M.; Yu, D.; Li, W.; Zhang, D.; Lu, F.; Gong, Q.-M.; Liu, F.; Jiang, J.-H.; Zheng, M.; et al. Serum $\mathrm{WFA}^{+}$-M2BP levels for evaluation of early stages of liver fibrosis in patients with chronic hepatitis B virus infection. Liver Int. 2016, 37, 35-44. [CrossRef]

26. Nishikawa, H.; Enomoto, H.; Iwata, Y.; Hasegawa, K.; Nakano, C.; Takata, R.; Nishimura, T.; Kazunori, Y.; Aizawa, N.; Sakai, Y.; et al. Impact of serum Wisteria floribunda agglutinin-positive Mac-2-binding protein and serum interferon-?-inducible protein-10 in primary biliary cirrhosis. Hepatol. Res. 2015, 46, 575-583. [CrossRef]

27. Nishikawa, H.; Enomoto, H.; Iwata, Y.; Hasegawa, K.; Nakano, C.; Takata, R.; Nishimura, T.; Yoh, K.; Aizawa, N.; Sakai, Y.; et al. Clinical significance of serum Wisteria floribunda agglutinin positive Mac-2-binding protein level and high-sensitivity C-reactive protein concentration in autoimmune hepatitis. Hepatol. Res. 2015, 46, 613-621. [CrossRef]

28. Kamada, Y.; Ono, M.; Hyogo, H.; Fujii, H.; Sumida, Y.; Yamada, M.; Mori, K.; Tanaka, S.; Maekawa, T.; Ebisutani, Y.; et al. Use of Mac-2 binding protein as a biomarker for nonalcoholic fatty liver disease diagnosis. Hepatol. Commun. 2017, 1, 780-791. [CrossRef]

29. Åberg, F.; Helenius-Hietala, J.; Puukka, P.; Färkkilä, M.; Jula, A. Interaction between alcohol consumption and metabolic syndrome in predicting severe liver disease in the general population. Hepatology 2018, 67, 2141-2149. [CrossRef]

30. Åberg, F.; Färkkilä, M. Drinking and Obesity: Alcoholic Liver Disease/Nonalcoholic Fatty Liver Disease Interactions. Semin. Liver Dis. 2020, 40, 154-162. [CrossRef]

31. Eslam, M.; Newsome, P.N.; Sarin, S.K.; Anstee, Q.M.; Targher, G.; Gomez, M.R.; Zelber-Sagi, S.; Wong, V.W.-S.; Dufour, J.-F.; Schattenberg, J.M.; et al. A new definition for metabolic dysfunction-associated fatty liver disease: An international expert consensus statement. J. Hepatol. 2020. [CrossRef] [PubMed]

32. Saadeh, S.; Younossi, Z.M.; Remer, E.M.; Gramlich, T.; Ong, J.P.; Hurley, M.; Mullen, K.D.; Cooper, J.N.; Sheridan, M.J. The utility of radiological imaging in nonalcoholic fatty liver disease. Gastroenterology 2002, 123, 745-750. [CrossRef] [PubMed]

33. Hamano, M.; Kamada, Y.; Kiso, S.; Furuta, K.; Kizu, T.; Chatani, N.; Egawa, M.; Takemura, T.; Ezaki, H.; Yoshida, Y.; et al. Adiponectin negatively correlates with alcoholic and non-alcoholic liver dysfunction: Health check-up study of Japanese men. Hepatol. Res. 2012, 43, 238-248. [CrossRef] [PubMed]

34. Sterling, R.K.; Lissen, E.; Clumeck, N.; Sola, R.; Correa, M.C.; Montaner, J.; Sulkowski, M.S.; Torriani, F.J.; Dieterich, D.T.; Thomas, D.L.; et al. Development of a simple noninvasive index to predict significant fibrosis in patients with HIV/HCV coinfection. Hepatology 2006, 43, 1317-1325. [CrossRef] [PubMed] 
35. Shah, A.G.; Lydecker, A.; Murray, K.; Tetri, B.N.; Contos, M.J.; Sanyal, A.J. NASH Clinical Research Network Comparison of Noninvasive Markers of Fibrosis in Patients with Nonalcoholic Fatty Liver Disease. Clin. Gastroenterol. Hepatol. 2009, 7, 1104-1112. [CrossRef]

36. Kamada, Y.; Miyoshi, E. Mac-2 binding protein is a useful liver fibrosis biomarker for NAFLD/NASH. Trends Glycosci. Glycotechnol. 2019, 29, E85-E92. [CrossRef]

37. Yoneda, M.; Fujii, H.; Sumida, Y.; Hyogo, H.; Itoh, Y.; Ono, M.; Eguchi, Y.; Suzuki, Y.; Aoki, N.; Kanemasa, K.; et al. Platelet count for predicting fibrosis in nonalcoholic fatty liver disease. J. Gastroenterol. 2011, 46, 1300-1306. [CrossRef]

38. Sumida, Y.; Yoneda, M.; Hyogo, H.; Itoh, Y.; Ono, M.; Fujii, H.; Eguchi, Y.; Suzuki, Y.; Aoki, N.; Kanemasa, K.; et al. Validation of the FIB4 index in a Japanese nonalcoholic fatty liver disease population. BMC Gastroenterol. 2012, 12, 2. [CrossRef]

39. Cheung, A.; Figueredo, C.; Rinella, M.E. Nonalcoholic Fatty Liver Disease. Am. J. Gastroenterol. 2019, 114, 579-590. [CrossRef]

40. Srivastava, A.; Gailer, R.; Tanwar, S.; Trembling, P.; Parkes, J.; Rodger, A.; Suri, D.; Thorburn, U.; Sennett, K.; Morgan, S.; et al. Prospective evaluation of a primary care referral pathway for patients with non-alcoholic fatty liver disease. J. Hepatol. 2019, 71,371-378. [CrossRef]

41. Iwata, A.; Kamada, Y.; Ebisutani, Y.; Yamamoto, A.; Ueda, Y.; Arai, H.; Fujii, H.; Takamatsu, S.; Maruyama, N.; Maeda, M.; et al. Establishment of mouse Mac-2 binding protein enzyme-linked immunosorbent assay and its application for mouse chronic liver disease models. Hepatol. Res. 2016, 47, 902-909. [CrossRef] [PubMed]

42. Resnick, D.; Pearson, A.; Krieger, M. The SRCR superfamily: A family reminiscent of the Ig superfamily. Trends Biochem. Sci. 1994, 19, 5-8. [CrossRef]

43. Trahey, M.; Weissman, I.L. Cyclophilin C-associated protein: A normal secreted glycoprotein that down-modulates endotoxin and proinflammatory responses in vivo. Proc. Natl. Acad. Sci. USA 1999, 96, 3006-3011. [CrossRef] [PubMed]

44. Umemura, T.; Joshita, S.; Sekiguchi, T.; Usami, Y.; Shibata, S.; Kimura, T.; Komatsu, M.; Matsumoto, A.; Ota, M.; Tanaka, E. Serum Wisteria floribunda Agglutinin-Positive Mac-2-Binding Protein Level Predicts Liver Fibrosis and Prognosis in Primary Biliary Cirrhosis. Am. J. Gastroenterol. 2015, 110, 857-864. [CrossRef] [PubMed]

(C) 2020 by the authors. Licensee MDPI, Basel, Switzerland. This article is an open access article distributed under the terms and conditions of the Creative Commons Attribution (CC BY) license (http://creativecommons.org/licenses/by/4.0/). 